OPEN ACCESS

Edited by:

Chandrashekhar Pralhad Joshi, Michigan Technological University,

United States

Reviewed by:

Nookaraju Akula,

Kaveri Seed Company Ltd., India

Anindya Ganguly,

Washington University in St. Louis,

United States

*Correspondence:

Lei Shan

shlei1025@sina.com

Shubo Wan

wansb@saas.ac.cn

Specialty section:

This article was submitted to

Plant Biotechnology,

a section of the journal

Frontiers in Plant Science

Received: 04 January 2018

Accepted: 14 February 2018

Published: 06 March 2018

Citation

Tang G, Xu P, Ma W, Wang F, Liu Z,

Wan S and Shan L (2018)

Seed-Specific Expression of AtLEC1

Increased Oil Content and Altered Fatty Acid Composition in Seeds of Peanut (Arachis hypogaea L.).

Front. Plant Sci. 9:260.

doi: 10.3389/fpls.2018.00260

\section{Seed-Specific Expression of AtLEC1 Increased Oil Content and Altered Fatty Acid Composition in Seeds of Peanut (Arachis hypogaea L.)}

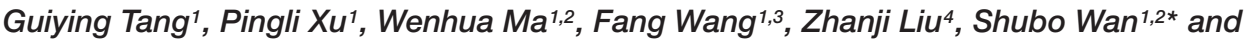 \\ Lei Shan ${ }^{1,2,3 *}$ \\ ${ }^{1}$ Shandong Provincial Key Laboratory of Crop Genetic Improvement, Ecology and Physiology, Bio-Tech Research Center, \\ Shandong Academy of Agricultural Sciences, Jinan, China, ${ }^{2}$ College of Life Sciences, Shandong University, Jinan, China, \\ ${ }^{3}$ College of Life Sciences, Shandong Normal University, Jinan, China, ${ }^{4}$ Shandong Cotton Research Center, Shandong \\ Academy of Agricultural Sciences, Jinan, China
}

Peanut (Arachis hypogaea L.) is one of the major oil crops and is the fifth largest source of plant oils in the world. Numerous genes participate in regulating the biosynthesis and accumulation of the storage lipids in seeds or other reservoir organs, among which several transcription factors, such as LEAFY COTYLEDON1 (AtLEC1), LEC2, and WRINKLED1 (WR/1), involved in embryo development also control the lipid reservoir in seeds. In this study, the AtLEC1 gene was transferred into the peanut genome and expressed in a seed-specific manner driven by the NapinA full-length promoter or its truncated 230-bp promoter. Four homozygous transgenic lines, two lines with the longer promoter and the other two with the truncated one, were selected for further analysis. The AtLEC1 mRNA level and the corresponding protein accumulation in different transgenic overexpression lines were altered, and the transgenic plants grew and developed normally without any detrimental effects on major agronomic traits. In the developing seeds of transgenic peanuts, the mRNA levels of a series of genes were upregulated. These genes are associated with fatty acid (FA) biosynthesis and lipid accumulation. The former set of genes included the homomeric ACCase A (AhACC II), the $\mathrm{BC}$ subunit of heteromeric ACCase (AhBC4), ketoacyl-ACP synthetase (AhKAS II), and stearoyl-ACP desaturase (AhSAD), while the latter ones were the diacylglycerol acyltransferases and oleosins (AhDGAT1, AhDGAT2, AhOle1, AhOle2, and AhOle3). The oil content and seed weight increased by $4.42-15.89 \%$ and $11.1-22.2 \%$, respectively, and the levels of major FA components including stearic acid, oleic acid, and linoleic acid changed significantly in all different lines.

\footnotetext{
Keywords: transgenic peanut (Arachis hypogaea L.), Arabidopsis LEC1 (AtLEC1) overexpression, seed-specific promoter, fatty acid synthesis, oil accumulation
}

\section{INTRODUCTION}

Peanut (Arachis hypogaea L.) is the fifth largest source of plant oil in the world, after soybean, rapeseed, cotton, and sunflower (de Paula et al., 2017). Its global production during 2015-2016 and 2016-2017 was 40.42 and 42.77 million tons, respectively (USDA, 2018). The oil contents of its seeds in the current commercial cultivars account for about 50\% of seed fried weight (Li et al., 2009; 
Chi et al., 2014). In recent years, as the demand for plant oils has sharply increased, improving the oil content and quality of oilseed crops has become a major goal globally.

Storage lipids, which mainly consist of triacylglycerol (TAG) synthesized from glycerol-3-phosphate (G3P) and fatty acids (FAs), generally accumulate during the maturation of seeds and are the major energy source for younger seedlings. In recent decades, many genes encoding key FA biosynthesis- or lipid reservoir-related enzymes or enzyme subunits from different organisms have been transformed into plants and increased the levels of lipids in transgenic seeds to varying degrees (Shorrosh et al., 1995; Zou et al., 1997; Jako et al., 2001). However, in some cases the oil content was reduced, for example, by overexpressing the gene encoding spinach 3-ketoacyl-ACP synthase III (KAS III) in transgenic rapeseed seeds (Dehesh et al., 2001). Nevertheless, FA synthesis and accumulation involve a highly coordinated regulatory network, not only associated with FA synthesis and lipid accumulation genes, but also some glycolysis related genes during carbon metabolism (Broun et al., 1999; Thelen and Ohlrogge, 2002; Jaworski and Cahoon, 2003; Cahoon et al., 2007; Weselake et al., 2009). Thus, manipulating only one or a few genes in the pathway of FA synthesis and metabolism sometimes resulted in discordant changes of FA composition and lipid contents.

Some recent studies found that several genes encoding embryo-specific transcription factors associated with embryogenesis and development, including $L E A F Y$ COTYLEDON1 (AtLEC1), LEC1-LIKE (AtL1L), LEC2, and WRINKLED1 (WRI1), could regulate the coexpression of certain genes encoding key enzymes involved in FA biosynthesis and TAG accumulation in seeds (Focks and Benning, 1998; Lotan et al., 1998; Stone et al., 2001; Kwong et al., 2003; Cernac and Benning, 2004; Mu et al., 2008; Peng and Weselake, 2011). The highly conserved CCAAT motifs for LEC1 (or L1L) binding are significantly enriched in promoters of all FA biosynthesis-related genes (Peng and Weselake, 2011). Overexpression of these genes increases the FA contents in the transgenic plants, accompanied by the enhanced expression of key FA synthetic genes and glycolytic genes (Cernac and Benning, 2004; Baud et al., 2007; Mu et al., 2008; Andrianov et al., 2010; Tan et al., 2011; Manan et al., 2017). However, overexpression of these genes via their constitutive expression or expression at a high level in seeds always resulted in deleterious traits or undesirable pleiotropic effects. For example, ectopic expression of the AtLEC1 gene in the leaves of 35S:LEC1 transgenic Arabidopsis induced the formation of an embryo-like structure and caused developmental abnormalities (Lotan et al., 1998). Moreover, the seed-specific expression of ZmLEC1 under the control of the EARLY EMBRYO PROTEIN (EAP1) promoter significantly increased the embryo oil content of the resulting transgenic maize, but severely affected seed germination and leaf development of the transgenic plants. However, overexpression of $Z m W R I 1$, a WRI1-like gene in maize (Zea mays) seeds, increased the oil content without producing detrimental agronomic traits (Shen et al., 2010). Furthermore, a recent study showed that seed-specific expression of BnLEC1 and BnL1L, driven by two modified Napin A (NapA) promoters that only have 18 and $4 \%$ of the full-length promoter activity, respectively, significantly enhanced oil accumulation in transgenic rapeseed seeds without producing any detectable abnormalities (Tan et al., 2011).

In peanut breeding, key goals have long been to increase the oil content and improve the FA composition in peanut seeds. In this paper, we describe that, under the control of full-length or 230bp NapA promoter, transgenic peanuts exhibiting seed-specific expression of AtLEC1 grew and developed normally without any detrimental effects on major agronomic traits. In the developing seeds of the transgenic peanuts, the mRNA levels of a series of genes associated with FA biosynthesis and lipid accumulation were upregulated, the oil content and seed weight increased significantly, and the levels of major FAs changed markedly in all lines. These results provide an efficient approach for the genetic improvement of peanut seed oil production.

\section{MATERIALS AND METHODS}

\section{Plant Materials and Growth Conditions}

The peanut cultivar "Fenghua No. 1" (FH1), as the subject of transformation, was kept and reproduced by our group. The FH1 plants and all corresponding transgenic plants were grown in our experimental field for transgenic research. Under the field conditions, seeds were usually sown in early May and harvested in late September. When grown on culture medium, all seeds were completely immersed in water for $30 \mathrm{~min}$ and then surfacesterilized with $75 \%$ ethanol for $1 \mathrm{~min}$, followed by $0.1 \%$ mercury chloride for $15 \mathrm{~min}$, and then rinsed four times with sterile water. The sterilized seeds were sown on $1 / 2 \mathrm{MS}_{0}$ medium with $0.6 \%$ agar (Murashige and Skoog, 1962) and then cultured at $25^{\circ} \mathrm{C}$ under a $16 \mathrm{~h} / 8 \mathrm{~h}$ light/dark cycle.

\section{Construction of Plant Expression Vectors}

The plasmids pC2300-NapAF:AtLEC1(pF:AtLEC1) and pC2300NapA230:AtLEC1(p230:AtLEC1) (Figure 1) were kindly provided by Professor Jianru Zuo of the Institute of Genetics and Developmental Biology, Chinese Academy of Sciences. They are based on the pCAMBIA2300 framework with a NEOMYCIN PHOSPHOTRANSFERASE II (NPTII) gene for the selection of transformed plants, as well as the AtLEC1 gene driven by a 1101-bp (full-length) or 230-bp promoter of NapA from Brassica napus (B. napus), respectively. To facilitate the identification of foreign gene expression, a polypeptide tag of six $\times$ Myc was added at the C-terminus of AtLEC1.

\section{Generation of Transgenic Lines}

The two plasmids mentioned above were transformed into Agrobacterium tumefaciens strain LBA4404, which was then used for peanut transformation. Using the epicotyl fragments of FH1 seeds germinated for 4 days as explants, transformation of peanut plants was carried out by an Agrobacterium-mediated method. A single colony of Agrobacterium harboring the target plasmid was propagated in $100 \mathrm{~mL}$ of liquid YEP medium containing $50 \mathrm{mg} / \mathrm{L}$ kanamycin at $28^{\circ} \mathrm{C}$ on a shaker for $24-36 \mathrm{~h}$ and reached the logarithmic growth phase $\left(A_{600}\right.$ at $\left.0.8-1.0\right)$, and the bacterial cells were resuspended in an equal volume of 




FIGURE 1 | Structures of plant expression vectors harboring the foreign AtLEC1 gene driven by Napin A full-length or 230-bp promoter.

liquid medium for shoot induction [MIS; MS medium plus 8 $\mathrm{mg} / \mathrm{L}$ 6-benzylaminopurine (6-BAP), $0.7 \mathrm{mg} / \mathrm{L} \alpha$-naphthylacetic acid (NAA), $30 \mathrm{~g} / \mathrm{L}$ sucrose, $\mathrm{pH}$ 5.8]. The fresh explants were immersed in the suspension for $20 \mathrm{~min}$, blotted dry on sterile filter paper to remove excess bacteria, and then transferred onto MIS solid medium and cultured in the dark at $25 \pm 1^{\circ} \mathrm{C}$ for $48 \mathrm{~h}$. The infected epicotyl explants were washed with MIS liquid medium containing $500 \mathrm{mg} / \mathrm{L}$ carbenicillin and cultivated on the selection MIS medium containing $50 \mathrm{mg} / \mathrm{L}$ kanamycin and $500 \mathrm{mg} / \mathrm{L}$ carbenicillin. After culture for 20-30 days, the induced adventitious buds were excised from the primary explants and subcultured on MSE medium for stem elongation $\left(\mathrm{MS}_{0}+2 \mathrm{mg} / \mathrm{L}\right.$ 6 -BAP $+1.0 \mathrm{mg} / \mathrm{L}$ gibberellin $\mathrm{GA}_{3}, 30 \mathrm{~g} / \mathrm{L}$ sucrose, $\mathrm{pH}$ 5.8) containing the same screener and antimicrobial. When the kanamycin-resistant shoots reached approximately $5-6 \mathrm{~cm}$ in length, they were separated and grafted on the vigorous seedlings of wild-type peanut in a greenhouse.

The transgenic plants of the $\mathrm{T}_{0}$ generation were detected by polymerase chain reaction (PCR) and the sequencing of PCR products. Then, the positive transgenic plants of the $\mathrm{T}_{1}-\mathrm{T}_{3}$ generations were confirmed by PCR and at least 20 plants per line were detected. Using chi-squared test, the ratios of PCR-positive numbers to -negative numbers in every detected transgenic $T_{1}$ line were assessed, the lines in accordance with Mendel's first law were selected for generation, and the transgenic $T_{2}$ and $T_{3}$ progeny with positive PCR products were identified as homozygous lines to be used for further analysis. The PCR reaction conditions were as follows. The reaction mixture was predenatured for $5 \mathrm{~min}$ at $94^{\circ} \mathrm{C}$, and then amplified for 35 cycles by denaturing for $25 \mathrm{~s}$ at $94^{\circ} \mathrm{C}$, annealing for $30 \mathrm{~s}$ at $56^{\circ} \mathrm{C}$, and extending for $45 \mathrm{~s}$ at $72^{\circ} \mathrm{C}$, followed by final extension for another $5 \mathrm{~min}$ at $72^{\circ} \mathrm{C}$. The forward and reverse primers for PCR were designed to match sequences in the promoter region and the ORF region of the target gene, respectively, with the following sequences: $5^{\prime}$-GTGATCGCCATGCAAATCTCC- $3^{\prime}$ and 5'-CCAAGCTTGCTCATAGCCCA-3'.

\section{Protein Extraction and Western Blotting}

Total soluble protein was extracted from the seeds at 3040 days after pegging (DAP) of control FH1 and the transgenic $\mathrm{T}_{3}$ generation lines using a plant total protein extraction kit (Sangon Biotech Co., Ltd., Shanghai, China). Total protein extracts of over $40-50 \mu \mathrm{g}$ for each sample were boiled for 5 min in $6 \times$ loading buffer (Transgen Biotech, Beijing, China), separated by $10 \%$ sodium dodecyl sulfate - polyacrylamide gel electrophoresis (SDS-PAGE), and then transferred to a $0.45-\mu \mathrm{m}$ polyvinylidene difluoride (Roche Applied Science, Mannheim, Germany) membrane by the wet electrotransfer method (Tovey and Baldo, 1987). After transfer, blotting efficiency was checked by reversibly staining the transferred proteins with Ponceau $S$ solution. Using mouse monoclonal antibody Myc-Tag (9B11; Cell Signaling Technology, Inc.) as the primary antibody, western blotting was carried out in accordance with the instructions of the Lumi-Light ${ }^{\text {PLUS }}$ Western Blotting Kit (Roche Applied Science).

\section{Analysis of Gene Expression by qRT-PCR}

Total RNA was isolated from the immature seeds at about 15,30 , and 60 DAP for the control and transgenic plants by an improved version of the cetyltrimethylammonium bromide method (Chen et al., 2011). The first-strand cDNA synthesis was undertaken using oligo(dT) as a primer, in accordance with the instructions of the PrimeScript II 1st strand cDNA Synthesis Kit (Takara Biotechnology, Dalian, China). For realtime quantitative reverse transcription PCR (qRT-PCR) analysis, a diluted form of the RT reaction was used as a template and the amplification system was established with reference to the instructions of SYBR Premix Ex Taq (Tli RNaseH Plus; Takara Biotechnology, Dalian, China). The amplification was performed by predenaturation for $5 \mathrm{~min}$ at $94^{\circ} \mathrm{C}$, and then 40 cycles of $15 \mathrm{~s}$ at $94^{\circ} \mathrm{C}$ and $30 \mathrm{~s}$ at $60^{\circ} \mathrm{C}$. The relative expression levels of the target genes were analyzed with AhACTIN7 as an internal control and calculated using the formulas $F=2^{-\Delta \Delta C t}$ and $\Delta \Delta \mathrm{Ct}=(\mathrm{Ct}$ mean value of the target gene in the sample $-\mathrm{Ct}$ mean value of the housekeeping gene in the sample) $-(\mathrm{Ct}$ mean value of the target gene in the control - Ct mean value of the housekeeping gene in the control). The detected genes and all corresponding primers are listed in Supplementary Table S1.

\section{Analysis of Seed Lipid Content and Fatty Acid Composition}

The seed oil content was analyzed by the near-infrared reflectance (NIR) spectral method and the Soxhlet extraction method. NIR analysis of the oil content was carried out using a DA7250 NIR Analyzer (Perten Instruments, Hägersten, Sweden). The intact seeds from a single plant were scanned and at least six plants of each transgenic line and of the control were investigated.

For the Soxhlet extraction, over $2 \mathrm{~g}$ of seeds were ovendried for $2 \mathrm{~h}$ at $105^{\circ} \mathrm{C}$ and then cooled to room temperature. The dried seeds were ground into a fine powder and then transferred into a preweighed bag made of Whatman $3 \mathrm{M}$ 
filter papers (weight of filters and absorbent cotton recorded as M1), sealed, and weighed (M2). The bagged sample was transferred into a Soxhlet tube and extracted with about $100 \mathrm{~mL}$ of petroleum ether (boiling point $30-60^{\circ} \mathrm{C}$ ) using Soxhlet Extractor R306 (Behr, Germany) for over 25 cycles. After the extraction, the bagged sample was dried in a preweighed round-bottomed flask (M3) at $80^{\circ} \mathrm{C}$ to evaporate the remaining petroleum ether, cooled, and weighed (M4). The oil content was calculated using the following formula: $M_{\mathrm{r}}=\frac{M_{4}-M_{3}}{M_{2}-M_{1}} \times 100 \%$ (Shivani et al., 2011). For each transgenic and control plant, the mean values of triplicate samples were calculated.

Analysis of FA content and composition was performed by gas chromatography (GC) using an HP-6890 (Agilent Technologies, Santa Clara, CA, United States) equipped with a capillary bonded fused-silica column, DB-23 $(60 \mathrm{~m} \times 250 \mu \mathrm{m} \times 0.25 \mu \mathrm{m}$; J\&W Scientific, Rancho Cordova, CA, United States). The sample was treated as previously described (Tang et al., 2012). Each FA methyl ester (FAME) sample was analyzed three times. The analytical conditions were as follows: the initial column temperature was $180^{\circ} \mathrm{C}$, which was subsequently increased by a set program. The amount of added sample was $1 \mu \mathrm{L}$. The injector and detector temperatures were 260 and $270^{\circ} \mathrm{C}$, respectively, the $\mathrm{N}_{2}$ gas flow rate was $2.0 \mathrm{~mL}$ $\min ^{-1}$, and the split ratio was 30:1. FA content was calculated as absolute content (mg/g) using the GC area, based on the quantification of known amounts of internal standards. The quantities of the FAMEs in each sample were calculated using the following formula: $W_{\mathrm{i}}=A_{\mathrm{i}} M_{s} / A_{s} M$, where $M_{s}$ is the weight of the internal standard mixed into a sample, $A_{i}$ is the area of the individual FAME, $A_{s}$ is the area of the corresponding FAME in the internal standard, and $M$ is the sample weight.

\section{Investigation of Agronomic Traits}

The transgenic lines and their untransformed control were planted in adjacent fields. The seedling rate, plant height, branch number per plant, and flowering time were investigated during the vegetative development stage. Other traits including the number of pods per plant, the average pod and seed weights, pod shape, seed shape, and seed coat color were evaluated after harvest.

\section{Statistical Analysis}

All experiments were performed with three replicates. Student's $t$-test in SPSS (Statistical Product and Service Solutions) was applied to identify the differences between the transgenic lines and their control in oil content, seed and pod weight, and FA content, and $p<0.05$ and $p<0.01$ were considered to indicate significant differences. One-way analysis of variance (ANOVA) for gene expression levels among different transgenic peanut lines and their control at different developmental stages was performed by SPSS software, and the significance of differences $(p<0.05)$ was assessed by Duncan's new multiple range test with values with different letters being significantly different.

\section{RESULTS}

\section{Generation and Characterization of Transgenic Peanut Plants Carrying AtLEC1 Gene}

Two expression constructs, p230:AtLEC1 and pF:AtLEC1, were transformed into the peanut cultivar FH1 by A. tumefaciensmediated transformation. Multiple independent transformed plants with kanamycin resistance were obtained. Specific bands of $\sim 500$ bp including 63-bp Napin A promoter fragment and of $\sim 450$ bp including the AtLEC1 segment were observed upon PCR analysis of the transgenic plants, whereas the untransformed control showed no amplification (Figure 2). The PCR products were confirmed by sequencing. A total of 15 transgenic plants of the $T_{0}$ generation were obtained.

Out of $15 \mathrm{~T}_{0}$ plants, only 12 yielded seeds, which were used to produce the next generation. The positive transgenic plants of the $T_{1}-T_{3}$ generations were confirmed by PCR. Nine $T_{1}$ plants carrying the p230:AtLEC1 construct and $12 \mathrm{~T}_{1}$ plants harboring the pFL:AtLEC1 construct were yielded and used to create $T_{2}$ progeny. According to the chi-square test, the $T_{2}$ lines were all in accordance with the segregation ratio of Mendel's first law. Thus, $\mathrm{T}_{2}$ transgenic lines with single-copy insertion were obtained. These transgenic lines were derived from six transformation events (three events 030801, 031001, and 031607 with the 230-bp promoter, and another three events 040201, 040305, and 040603 with the full-length promoter) and planted in a greenhouse. The plants that were developing well and contained foreign genes were selected and then planted in a greenhouse to generate $\mathrm{T}_{3}$ homozygous transgenic lines. Four homozygous transgenic lines (030801-3-5, 031001-2-3, 04020111-8, and 040603-3-9) from two transformed constructs were used for further analysis.

\section{Analysis of Foreign Gene Expression by qRT-PCR and Western Blotting}

To check the transcription level of AtLEC1 in different transgenic lines, qRT-PCR analysis was performed by the SYBR green method using the specific primers for AtLEC1. The results showed that the expression levels of AtLEC1 in seeds differed among the different transgenic lines, and the accumulation of mRNA in the lines with the FL NapA promoter was higher than for those with the 230-bp promoter (Figure 3), suggesting that the FL promoter had higher transcriptional activity than the truncated one.

To evaluate the protein content in the seeds of transgenic plants containing AtLEC1, total soluble proteins were extracted from about $0.5 \mathrm{mg}$ of immature seeds at 30-40 DAP, and 40-50 $\mu \mathrm{g}$ of seed protein of each transgenic plant was separated by SDS-PAGE and used for western blotting. It was predicted that the molecular weight of the fusion protein of AtLEC1 and Myc tag is about $32 \mathrm{kDa}$. Using the Myc-Tag monoclonal antibody from mouse as the primary antibody, in western blotting, distinctive hybridization signals were detectable in the seeds of different transformed plants (Figure 4). 




FIGURE 2 | Polymerase chain reaction (PCR) amplification of the foreign fragment in transgenic peanut plants and untransformed control. M, DL2000 DNA markers (Takara); 1, untransformed control; 2-17, PCR products from transgenic plants.

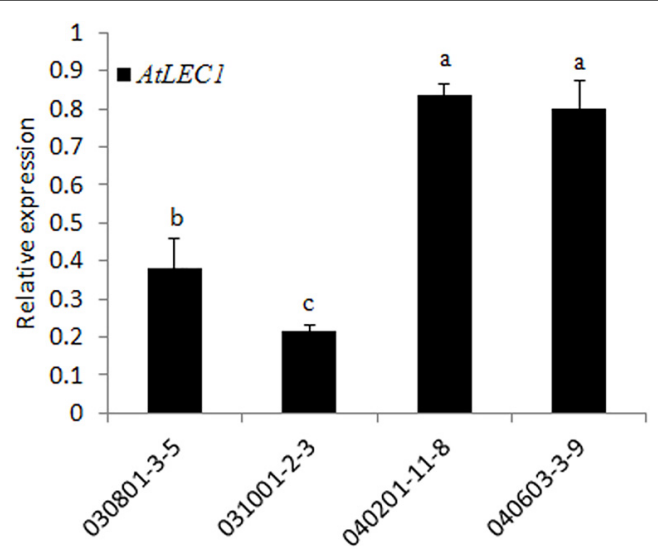

FIGURE 3 | Transcript level analysis of the foreign AtLEC1 gene in different transgenic peanut lines by qRT-PCR. One-way analysis of variance (ANOVA) for gene expression levels between different transgenic peanut lines was performed by SPSS software, and the significance of differences $(p<0.05)$ was assessed by Duncan's new multiple range test with values with different letters being significantly different.

\section{Analysis of Seed Oil Content and Weight}

Using the NIR method, the seed oil content and major FA composition of $21 \mathrm{~T}_{2}$ transgenic lines were analyzed. Compared with that of the non-transgenic FH1, 15 out of 21 analyzed transgenic lines had a remarkably enhanced seed oil level (data not shown). To measure the oil content precisely, four $\mathrm{T}_{3}$ transgenic lines (with each line representing a transformation event) with significant increases of oil content were selected and analyzed by the standard Soxhlet extraction method. Under the experimental conditions, the oil contents of the transgenic seeds ranged from 55.07 to $61.09 \%$, while that of nontransgenic control seeds was only $52.71 \%$, and they increased by $4.42-15.89 \%$ in comparison to the oil content of FH1 (Table 1).

Similar to the level in the control FH1, the transgenic lines produced 20-35 cocoon-shaped pods per plant, and over 90\% of them have two seeds per pod. There were not any visible changes in pod and seed size in transgenic peanuts compared to the untransformed control. A total of 20 full pods and 20 seeds were taken from each transgenic line and weighed to calculate the average weight per pod and per seed (Table 2). Compared with those in the control, the average weights of pods and seeds of the $\mathrm{T}_{3}$ transgenic lines increased by $2.9-14.9 \%$ and $11.1-22.2 \%$, respectively.

\section{Analysis of FA Contents and Composition in Transgenic Seeds}

The FA contents and composition of control FH1 and transgenic seeds were analyzed by GC. The results showed that, compared

TABLE 1 | Oil content in $T_{3}$ transgenic peanut seeds and $\mathrm{FH} 1$.

\begin{tabular}{lccc}
\hline Genotype & Line & $\begin{array}{c}\text { Oil content } \\
\text { (\% dry weight) }\end{array}$ & $\begin{array}{c}\text { Percentage of } \\
\text { increase }\end{array}$ \\
\hline WT & FH1 & $52.71 \pm 0.83$ & - \\
p230:AtLEC1 & $030801-3-5$ & $58.33 \pm 1.00^{* *}$ & 10.64 \\
& $031001-2-3$ & $55.07 \pm 0.53^{*}$ & 4.42 \\
pF:AtLEC1 & 040201-11-8 & $61.09 \pm 0.79^{* *}$ & 15.89 \\
& 040603-3-9 & $58.27 \pm 0.93^{* *}$ & 10.53
\end{tabular}

Mature seeds were used for the analysis of the oil content by the Soxhlet method. Asterisks indicated statistically significant differences compared with the control (Student's t-test: ${ }^{*} p<0.05,{ }^{* *} p<0.01$ ).

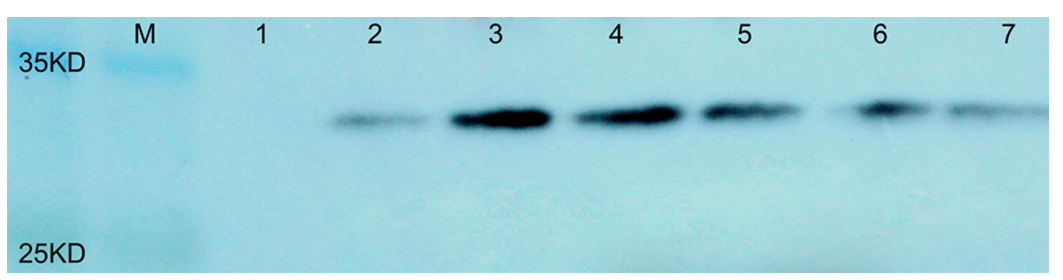

FIGURE 4 | Western blotting of the AtLEC1-MYC fusion protein in seeds of different transgenic peanut lines. M, prestained protein ladder (180, 135, 100, 75, 63, 48, 35, 25, 17, and $11 \mathrm{kDa}$; Thermo Scientific); 1, untransformed control; 2-7, different transgenic peanut lines (040305-6-5, 040201-11-8, 040603-3-9, 030801-3-5, 031001-2-3, and 031607-2-4, respectively). 
TABLE 2 | The Average weight of pods and seeds in $T_{3}$ transgenic peanut lines.

\begin{tabular}{|c|c|c|c|c|c|}
\hline Genotype & Line & $\begin{array}{l}\text { Average pod } \\
\text { weight (g) }\end{array}$ & $\begin{array}{c}\% \text { Increase over } \\
\text { control }\end{array}$ & $\begin{array}{c}\text { Average seed } \\
\text { weight }(g)\end{array}$ & $\begin{array}{c}\% \text { Increase over } \\
\text { control }\end{array}$ \\
\hline WT & $\mathrm{FH} 1$ & $2.42 \pm 0.08$ & - & $0.81 \pm 0.08$ & - \\
\hline \multirow[t]{2}{*}{ p230:AtLEC1 } & 030801-3-5 & $2.78 \pm 0.03^{* *}$ & 14.9 & $0.93 \pm 0.03^{* *}$ & 14.8 \\
\hline & 031001-2-3 & $2.49 \pm 0.02 *$ & 2.9 & $0.90 \pm 0.02^{* *}$ & 11.1 \\
\hline \multirow[t]{2}{*}{$\mathrm{pFL}: A t L E C 1$} & 040201-11-8 & $2.58 \pm 0.05^{*}$ & 6.6 & $0.92 \pm 0.05^{* *}$ & 13.6 \\
\hline & 040603-3-9 & $2.73 \pm 0.09^{* *}$ & 12.8 & $0.99 \pm 0.09^{* *}$ & 22.2 \\
\hline
\end{tabular}

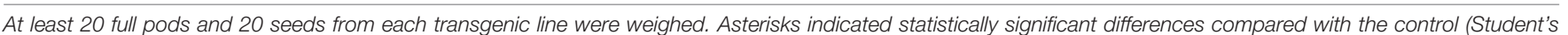
t-test: $\left.{ }^{*} P<0.05,{ }^{* *} P<0.01\right)$.

with their levels in FH1 seeds, FA C18:0 and C18:1n9 were significantly increased in transgenic seeds, whereas the C16:0 and C18:2n6 levels were significantly decreased. The most markedly changed FAs were unsaturated C18:1 and C18:2 in the transgenic lines 040201-11-8 and 040603-3-9 (Table 3). The greater increase of $\mathrm{C} 18: 1$ and decrease of $\mathrm{C} 18: 2$ resulted in elevation of the ratio of oleic acid (C18:1) to linoleic acid (C18:2, O/L) in these lines. Except in line 031001-2-3, the levels of the major long-chain FAs (C20 and longer chains) in transgenic lines were decreased to varying degrees (Table 3). Among those long-chain FAs, erucic acid (C22:1n9) is a key factor adversely affecting the quality of edible oil. Peanut cultivars including FH1 generally contain very low $\mathrm{C} 22: 1 \mathrm{n} 9$, and all transgenic lines similar to $\mathrm{FH} 1$ also have a low C22:1n9 level.

\section{Transcriptional Analysis of Several Genes Associated With FA Synthesis and Lipid Accumulation}

The majority of FA synthesis and lipid deposition in developing peanut seeds generally occurs around 10-70 DAP (data not shown). The full-length and 230-bp promoters of the NapA gene from $B$. napus were associated with increased expression from the heart stage to the cotyledon stage (10-30 days after pollination in rape) of seeds (Stålberg et al., 1993). Thus, we obtained immature seeds at three stages $(15,30$, and 60 DAP) as the materials for analysis and subjected them to qRT-PCR. The genes analyzed include the key genes in the FA de novo synthetic pathway [the genes encoding homomeric ACCase A (AhACC II), the BCCP subunit and $\mathrm{BC}$ subunit of heteromeric ACCase (AhBCCP1 and AhBC4), ketoacyl-ACP synthetase (AhKAS II), and the gene encoding stearoyl-ACP desaturase $(A h S A D)]$, as well as genes involved in lipid accumulation, such as those encoding diacylglycerol acyltransferase and oleosin (AhDGAT1, AhDGAT2, AhOle1, AhOle2, and AhOle3). Except for AhBCCP1, the expression of all genes mentioned above was enhanced to varying degrees in the seeds of transgenic lines at all three seed developmental stages (Figure 5). At the 15 DAP stage, compared with those in the untransformed control, the levels of the transcripts of these genes in seeds increased; their transcription levels were lower than those at other development stages, for both transgenic lines and controls. In comparison with the control, the mRNA abundance in the seeds of transgenic lines at 30 and 60 DAP was remarkably increased, and for some genes particularly at 30 DAP (Figure 5).

Owing to significant variation in the levels of C18:1, C18:2, and C16:0, we checked the transcript changes of $\Delta^{12} \mathrm{FA}$ desaturase (FAD2), and acyl-ACP thioesterases FATA and FATB related to the accumulation of the above FAs in the seeds of transgenic plants and the control at 30 DAP. It was found that the mRNA accumulation of FAD2 and FATA was decreased in the transgenic seeds with the two promoters of different lengths, while the transcript level of FATB increased in comparison with that in the control (Figure 6).

TABLE 3 | Comparison of FA composition and relative FA contents in mature seeds between FH1 and transgenic peanut lines (\%).

\begin{tabular}{|c|c|c|c|c|c|}
\hline \multirow[t]{2}{*}{ Fatty acid } & \multicolumn{5}{|c|}{ Line } \\
\hline & $030801-3-5$ & $031001-2-3$ & 040201-11-8 & 040603-3-9 & FH1 \\
\hline C16:0 & $12.44 \pm 0.00 *$ & $12.31 \pm 0.02^{*}$ & $10.12 \pm 0.03^{* *}$ & $10.26 \pm 0.00^{* *}$ & $13.04 \pm 0.06$ \\
\hline C16:1 & $0.05 \pm 0.00$ & $0.07 \pm 0.00$ & $0.06 \pm 0.00$ & $0.05 \pm 0.00$ & $0.07 \pm 0.00$ \\
\hline C18:0 & $3.44 \pm 0.01^{* *}$ & $3.51 \pm 0.00^{* *}$ & $4.05 \pm 0.01^{* *}$ & $4.00 \pm 0.04^{* *}$ & $2.93 \pm 0.01$ \\
\hline C18:1n9 & $40.48 \pm 0.02 *$ & $38.55 \pm 0.05^{*}$ & $52.06 \pm 0.01^{* *}$ & $49.65 \pm 0.00^{* *}$ & $37.3 \pm 0.03$ \\
\hline C18:2n6 & $37.98 \pm 0.03^{*}$ & $38.44 \pm 0.02^{*}$ & $28.89 \pm 0.02^{* *}$ & $30.91 \pm 0.10^{* *}$ & $40.62 \pm 0.12$ \\
\hline C18:3 & $0.04 \pm 0.00$ & $0.05 \pm 0.00$ & $0.04 \pm 0.00$ & $0.06 \pm 0.00$ & $0.06 \pm 0.00$ \\
\hline C2O:0 & $1.46 \pm 0.00$ & $1.49 \pm 0.00$ & $1.40 \pm 0.00$ & $1.43 \pm 0.01$ & $1.40 \pm 0.01$ \\
\hline C2O:1 & $0.68 \pm 0.00^{*}$ & $0.86 \pm 0.00^{* *}$ & $0.64 \pm 0.01^{* *}$ & $0.72 \pm 0.01$ & $0.76 \pm 0.00$ \\
\hline C22:1n9 & $0.03 \pm 0.00$ & $0.04 \pm 0.00$ & $0.03 \pm 0.00$ & $0.03 \pm 0.00$ & $0.04 \pm 0.00$ \\
\hline
\end{tabular}

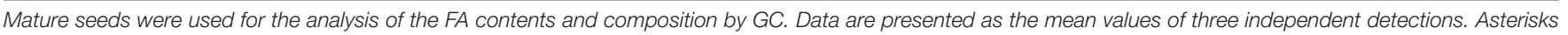
indicated statistically significant differences compared with the control (Student's t-test: ${ }^{*} p<0.05,{ }^{* *} p<0.01$ ). 




FIGURE 5 | Expression analysis of nine endogenous genes involved in FA synthesis and lipid accumulation in the developmental seeds of different transgenic peanut lines, as measured by qRT-PCR. One-way analysis of variance (ANOVA) for gene expression levels among different transgenic peanut lines and their control at different developmental stages was performed by SPSS software, and the significance of differences $(p<0.05)$ was assessed by Duncan's new multiple range test with values with different letters being significantly different. FH1, untransformed control. Relative transcript levels were quantified by comparison to that of peanut actin7 as a reference gene.

\section{DISCUSSION}

\section{AtLEC1 Overexpression in Peanut Seeds Improved the Oil Content and Weight of Seeds, While Not Altering the Major Agronomic Traits}

Improving the oil content of peanut seeds has consistently been a target of peanut breeding programs. Against this background, we transferred a key regulator of FA biosynthesis, the AtLEC1 gene, into peanut seeds and obtained several transgenic lines with higher oil content and heavier seeds than the untransformed control. These transgenic plants harboring the full-length or truncated 230-bp seed-specific NapA promoter did not show any visible developmental abnormalities regarding major agronomic traits. Previous studies indicated that the overexpression of some seed development-related genes, such as LEC1, LEC1like, and WRI1, via either constitutive expression or expression at a higher level in dicots and monocots could increase the oil content, but also led to a series of disorders of agronomic traits (Mu et al., 2008; Shen et al., 2010; Tan et al., 2011). For example, the overexpression of AtLEC1 by an estradiolinducible promoter (pER8) caused an elevated FA level in transgenic Arabidopsis seedlings. A similar phenotype was observed in transgenic Arabidopsis overexpressing LEC1-like genes of $B$. napus. However, when germinated and grown in the presence of estradiol, transgenic seedlings showed markedly reduced growth (Mu et al., 2008). Moreover, the overexpression of $Z m L E C 1$ under two promoters that preferentially function at the embryonic stage, a strong OLEOSIN (OLE) promoter and a weaker EARLY EMBRYO PROTEIN (EAP1) promoter, similarly increased the seed oil accumulation and embryo size, but caused reductions in seed germination and leaf growth (Shen et al., 2010). The undesirable phenotypes in transgenic lines produced by the strong OLE promoter were more serious than those by the weaker EAP1 promoter (Shen et al., 2010). In our study, two seed-specific promoters, namely, the fulllength 1101-bp NapA and the truncated 230-bp NapA, were used. The profiles of their expression reveal the characteristics of developmental dependence and spatial specificity, similar to those in embryos of transgenic tobacco, with expression being barely detectable 5-10 days after pollination (globule to heart stages), but with significant enhancement at 10-15 days (heart to torpedo stages), and reaching the highest expression level at 30 days (maturation stage) (Stålberg et al., 1993; Ellerström et al., 1996; Tan et al., 2011). The 1101-bp promoter was found to direct 


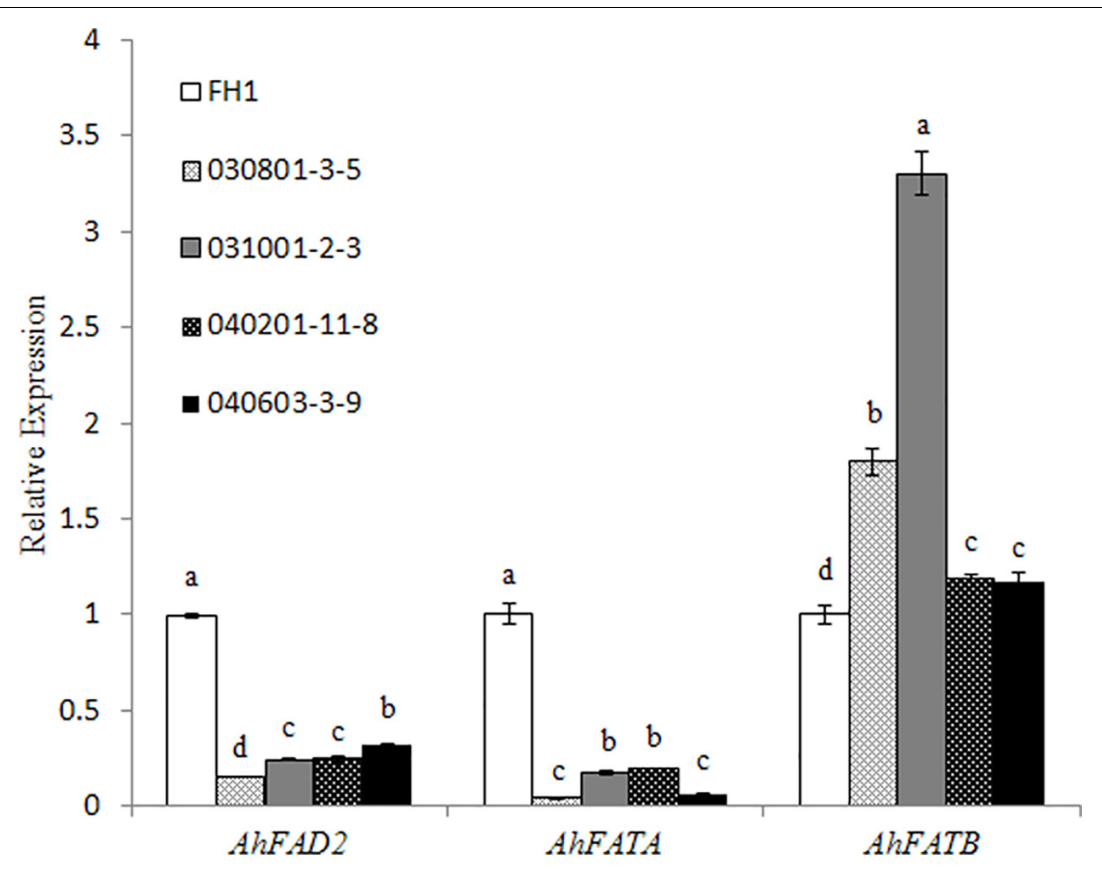

FIGURE 6 | Expression analysis of AhFAD2, AhFATA, and AhFATB in the seeds of different transgenic lines at 30 DAP by qRT-PCR. One-way analysis of variance (ANOVA) for gene expression levels among different transgenic peanut lines and their control was performed by SPSS software, and the significance of differences $(p<0.05)$ was assessed by Duncan's new multiple range test with values with different letters being significantly different FH1, untransformed control. Relative transcript levels were quantified by comparison to that of peanut actin7 as a reference gene.

$\beta$-GLUCURONIDASE (GUS) synthesis in the transgenic tobacco embryos at a level approximately 10 -fold higher than the $35 \mathrm{~S}$ promoter (Stålberg et al., 1993), and the 230-bp promoter lost more than $82 \%$ of the 1101-bp promoter's activity (Tan et al., 2011). We found that the expression patterns of all genes in the transgenic peanut seeds were basically consistent with the performance of the two promoters. That is, the transcript levels of most genes in transgenic 30- and 60-DAP seeds harboring the 1101-bp NapA promoter were higher than those with the 230-bp promoter. However, irrespective of whether the full-length NapA or 230-bp promoter was used, the transgenic lines exhibited normal traits similar to those of the control FH1 (Figure 7). Regarding these similarities to the agronomic characteristics of FH1, the height of these lines was also about $43 \mathrm{~cm}$, and they had inverted oval-shaped darker-green leaves, 9-10 branches of stems, and produced 20-35 cocoon-shaped pods in each plant. In addition, there were not any visible changes in pod and seed size in transgenic peanuts compared to $\mathrm{FH} 1$. In all transgenic peanut seeds, oil contents increased significantly, whereas protein contents remained unchanged. Therefore, it was suggested that the increase of seed weights was attributable to an enhancement of TAG accumulation. Thus, our results conflict with those of transgenic Arabidopsis reported by Tan et al., who found that transgenic plants overexpressing BnLEC1 controlled by the full-length NapA promoter exhibited severe abnormality after germination, and some plants died before flowering or did not produce seeds. Our results led to the conclusion that the FA biosynthesis-related genes in our AtLEC1 transgenic peanut plants with the 1101-bp promoter were reasonably upregulated.

\section{The Distinct Change of Main FA Contents in the Transgenic Seeds Is Due to Some Genes Involved in FA Modification and TAG Accumulation Expressed Synergistically}

In higher plants, the biosynthesis of FA and TAG has been well characterized by biochemical and molecular biology approaches. FA is synthesized de novo mainly from acetyl-CoA in plastids by a repeated series of reactions, including the carboxylation catalyzed by ACCase, and the continuous condensation, reduction, and dehydration reactions accompanied by the two-carbon chain length extension in each cycle, resulting in major acyl-ACPs such as 16:0-ACP, 18:0-ACP, and 18:1-ACP. Then, by the thioesterases FATA and FATB, the majority of acyl moieties are hydrolyzed from ACP and released into the acyl-CoA pool in the cytoplasm. Subsequently, the desaturation and elongation of FA acyl chains are performed by different FA desaturases (FADs) and elongases (FAEs) in plastid or in endoplasmic reticulum. Finally, acyl chains are added at the sn1-3 sites of the G3P backbone by a series of acyltransferases and form the TAG (Ohlrogge and Browse, 1995; Voelker and Kinney, 2001; Sasaki and Nagano, 2004). In recent years, several studies have indicated that TAG biosynthesis pathways include the acyl-CoA-dependent Kennedy pathway, the acyl-CoA-independent pathway, and a novel monoacylglycerol pathway (Yu et al., 2015). Thus, the FA composition and content in TAG not only rely on the components of the acyl-CoA pool, but are also involved in the network of acyl fluxes (Chen et al., 2015; Yu et al., 2015; Bates, 2016; Woodfield et al., 2017). 


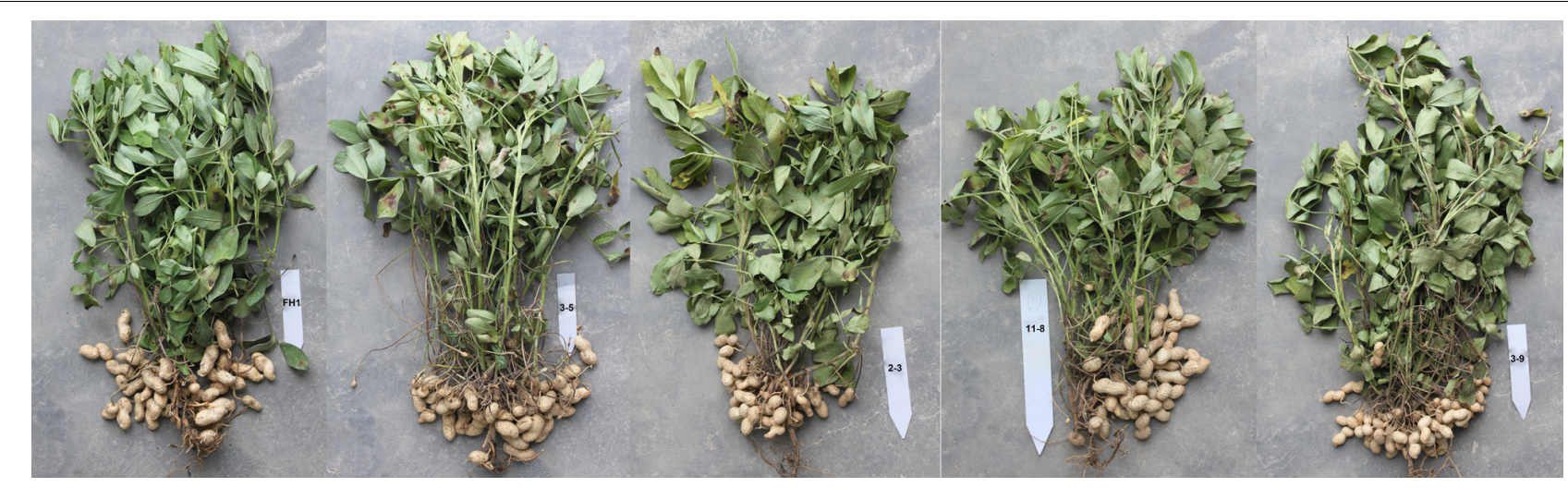

FIGURE 7 | Phenotype of transgenic peanut and its untransformed control at the harvest stage.

Substantial diversity in oil storage is observed in terms of the level of accumulation, the rate of oil synthesis, and the TAG structure in the reservoir tissues of different plant species, which is displayed in the assembly pathways of TAG and the different functions of corresponding enzymes (Peng and Weselake, 2011; Troncoso-Ponce et al., 2011; Yu et al., 2015). In Arabidopsis and B. napus, DGAT1 is the predominant enzyme in TAG synthesis, which catalyzes the accumulation of normal TAG with a high proportion of erucic acid (C22:1) in seeds, whereas in Ricinus communis, DGAT2 may be important for incorporation of the hydroxylated acyl into TAG (Zou et al., 1999; Troncoso-Ponce et al., 2011). Peanut seeds are enriched in oleic acid and linoleic acid, which account for $36-67 \%$ and $15-43 \%$ of the oil content of seeds, respectively. AhDGAT1 was considered to be the key enzyme for synthesizing TAG in seeds, which preferentially incorporates unsaturated C18 FAs into TAG (Chi et al., 2014; Zheng et al., 2017). In our transgenic peanuts overexpressing AtLEC1, compared with that in the control, AhDGAT1 expression was significantly enhanced at three developmental stages of seeds, resulting in a remarkable increase of the oil contents of transgenic seeds, where the C18 unsaturated FAs were the major components. In fact, lipid accumulation in seeds presents a range of distributions among the various tissue types, and the lipid profiles also have tissue or spatial specificity. The embryonic axis was particularly enriched in palmitic acid, while the cotyledon mainly accumulated more oleic acid (Horn et al., 2012, 2013; Woodfield et al., 2017). In our transgenic lines, the FL or 230bp Napin A promoter had higher activity in cotyledons prior to formation of the embryo axis (Stålberg et al., 1993; Ellerström et al., 1996). Thus, the C16 FAs in transgenic seeds accounted for a lower proportion of the total than in the control.

Woodfield et al. (2017) reported that the discrepant pattern of FA composition between the embryonic tissues and the cotyledons could be attributable to a spatial difference in the expression of FATA and FATB. In general, the thioesterase FatA has higher in vitro activity for 18:1-ACP and lower activities for 18:0-ACP and 16:0-ACP substrates, while FatB preferentially acts on saturated FAs containing 8-18 carbons (Salas and Ohlrogge, 2002; Wallis and Browse, 2002). In the fata mutant of Arabidopsis, a reduction of FATA was found to affect the ratio between C16 and C18 FAs, and caused a drop of palmitic acid incorporated into glycerolipids, but no apparent changes of C16 FA in seeds. In contrast, in Arabidopsis fatb mutant, the relative C16:0 FA content was significantly decreased in all tissues including seeds, and C18:0 content also decreased in seeds (Bonaventure et al., 2003 , 2004). In peanut, there were some differences in the profiles of substrates hydrolyzed by FATB. Ectopic expression of AhFATB1 in Escherichia coli effectively enhanced the levels of myristic acid (14:0), palmitoleic acid (16:1), and oleic acid $(18: 1)$, while the level of palmitic acid (16:0) decreased (Chen et al., 2012). In our results, the expression level of $A h S A D$ markedly increased in transgenic seeds, which catalyzed more 18:0-ACP molecules to form 18:1-ACP. In addition, the increase of AhFATB1 expression could release more 18:1-ACP molecules into the acyl-CoA pool and further participate in the synthesis of TAG. In addition, suppression of the AhFAD2 transcriptional level caused an increase of $18: 1$ by a reduction of the synthetic flux of 18:2. Therefore, the changes in the expression of these genes resulted in a significant increase of oleic acid (18:1) content in the seeds of all transgenic lines, and a corresponding reduction of the linoleic acid (18:2) content.

\section{CONCLUSION}

The overexpression of AtLEC1 in a seed-specific pattern in peanut increased the oil content and seed weight, and altered the FA composition of seeds by coordinately regulating the expression of several FA synthesis- and TAG accumulation-related genes. Moreover, there were no major changes in the main agronomic traits in all analyzed transgenic lines. This study provides a reasonable approach for the genetic improvement of peanut seed oil production.

\section{AUTHOR CONTRIBUTIONS}

LS and SW: conceived and designed the experiments. GT, PX, WM, and FW: performed the experiments. GT, PX, WM, ZL and LS: analyzed the data. GT, ZL, and LS: wrote the paper. All authors revised the draft and approved the final manuscript. 


\section{FUNDING}

This work was supported by the National Natural Science Foundation of China (30971546 and 31470349), Shandong Province Germplasm Innovation and Utilization Project.

\section{REFERENCES}

Andrianov, V., Borisjuk, N., Pogrebnyak, N., Brinker, A., Dixon, J., Spitsin, S., et al. (2010). Tobacco as a production platform for biofuel: overexpression of Arabidopsis DGAT and LEC2 genes increases accumulation and shifts the composition of lipids in green biomass. Plant Biotechnol. J. 8, 277-287. doi: 10.1111/j.1467-7652.2009.00458.x

Bates, P. D. (2016). Understanding the control of acyl flux through the lipid metabolic network of plant oil biosynthesis. Biochim. Biophys. Acta 1861(9 Pt B), 1214-1225. doi: 10.1016/j.bbalip.2016.03.021

Baud, S., Mendoza, M. S., To, A., Harscoet, E., Lepiniec, L., and Dubreucq, B. (2007). WRINKLED1 specifies the regulatory action of LEAFY COTYLEDON2 towards fatty acid metabolism during seed maturation in Arabidopsis. Plant J. 50, 825-838. doi: 10.1111/j.1365-313X.2007.03092.x

Bonaventure, G., Bao, X., Ohlrogge, J., and Pollard, M. (2004). Metabolic responses to the reduction in palmitate caused by disruption of the FATB gene in Arabidopsis. Plant Physiol. 135, 1269-1279. doi: 10.1104/pp.104. 043372

Bonaventure, G., Salas, J. J., Pollard, M., and Ohlrogge, J. (2003). Disruption of the FATB gene in Arabidopsis demonstrates an essential role of saturated fatty acids in plant growth. Plant Cell 15, 1020-1033. doi: 10.1105/tpc.008946

Broun, P., Gettner, S., and Somerville, C. (1999). Genetic engineering of plant lipids. Annu. Rev. Nutr. 19, 197-216. doi: 10.1146/annurev.nutr.19.1.197

Cahoon, E. B., Shockey, J. M., Dietrich, C. R., Gidda, S. K., Mullen, R. T., and Dyer J. M. (2007). Engineering oilseeds for sustainable production of industrial and nutritional feedstocks: solving bottlenecks in fatty acid flux. Curr. Opin. Plant Biol. 10, 236-244. doi: 10.1016/j.pbi.2007.04.005

Cernac, A., and Benning, C. (2004). WRINKLED1 encodes an AP2/EREB domain protein involved in the control of storage compound biosynthesis in Arabidopsis. Plant J. 40, 575-585. doi: 10.1111/j.1365-313X.2004.02235.x

Chen, G., He, Q. F., Xuan, N., Peng, Z. Y., Shan, L., Tang, G. Y., et al. (2012). Functional expression analysis of an acyl-ACP thioesterase FatB1 from Arachis hypogaea L. seeds in Escherichia coli. J. Food Agric. Environ. 10, 332-336.

Chen, G., Shan, L., Zhou, L. X., Tang, G. Y., and Bi, Y. P. (2011). The comparison of different methods for isolating total RNA from peanuts. Chin. Agric. Sci. Bull. 27, 214-218.

Chen, G., Woodfield, H. K., Pan, X., Harwood, J. L., and Weselake, R. J. (2015). Acyl-trafficking during plant oil accumulation. Lipids 50, 1057-1068. doi: 10.1007/s11745-015-4069-x

Chi, X., Hu, R., Zhang, X., Chen, M., Chen, N., Pan, L., et al. (2014). Cloning and functional analysis of three diacylglycerol acyltransferase genes from peanut (Arachis hypogaea L.). PLoS One 9:e105834. doi: 10.1371/journal.pone.0105834

de Paula, A. F., Dinato, N. B., Vigna, B. B. Z., and Fávero, A. P. (2017). Recombinants from the crosses between amphidiploid and cultivated peanut (Arachis hypogaea) for pest-resistance breeding programs. PLoS One 12:e0175940. doi: 10.1371/journal.pone.0175940

Dehesh, K., Tai, H., Edwards, P., Byrne, J., and Jaworski, J. G. (2001). Overexpression of 3-ketoacyl-acyl-carrier protein synthase IIIs in plants reduces the rate of lipid synthesis. Plant Physiol. 125, 1103-1114. doi: 10.1104/ pp.125.2.1103

Ellerström, M., Stålberg, K., Ezcurra, I., and Rask, L. (1996). Functional dissection of a napin gene promoter: identification of promoter elements required for embryo and endosperm-specific transcription. Plant Mol. Biol. 32, 1019-1027. doi: 10.1007/BF00041385

Focks, N., and Benning, C. (1998). wrinkled1: a novel, low-seed-oil mutant of Arabidopsis with a deficiency in the seed-specific regulation of carbohydrate metabolism. Plant Physiol. 118, 91-101. doi: 10.1104/pp.118.1.91

Horn, P. J., Korte, A. R., Neogi, P. B., Love, E., Fuchs, J., Strupat, K., et al. (2012). Spatial mapping of lipids at cellular resolution in embryos of cotton. Plant Cell 24, 622-636. doi: 10.1105/tpc.111.094581

\section{SUPPLEMENTARY MATERIAL}

The Supplementary Material for this article can be found online at: https://www.frontiersin.org/articles/10.3389/fpls.2018.00260/ full\#supplementary-material

Horn, P. J., Silva, J. E., Anderson, D., Fuchs, J., Borisjuk, L., Nazarenus, T. J., et al. (2013). Imaging heterogeneity of membrane and storage lipids in transgenic Camelina sativa seeds with altered fatty acid profiles. Plant J. 76, 138-150. doi: $10.1111 /$ tpj.12278

Jako, C., Kumar, A., Wei, Y., Zou, J., Barton, D. L., Giblin, E. M., et al. (2001). Seedspecific over-expression of an Arabidopsis cDNA encoding a diacylglycerol acyltransferase enhances seed oil content and seed weight. Plant Physiol. 126, 861-874. doi: 10.1104/pp.126.2.861

Jaworski, J., and Cahoon, E. B. (2003). Industrial oils from transgenic plants. Curr. Opin. Plant Biol. 6, 178-184. doi: 10.1016/S1369-5266(03)00013-X

Kwong, R. W., Bai, A. Q., Lee, H., Kwong, L. W., Fischer, R. L., Gordberg, R. B., et al. (2003). LEAFY COTYLEDON1-LIKE defines a class of regulators essential for embryo development. Plant Cell 15, 5-18. doi: 10.1105/tpc. 006973

Li, X. D., Chao, Y. L., Hu, Y. P., Xiao, L., Wu, Y. H., Wu, G., et al. (2009). Fatty acid accumulation pattern in developing seeds of peanut. Chin. J. Oil Crop Sci. 31, 157-162, 172

Lotan, T., Ohto, M., Yee, K. M., West, M. A., Lo, R., Kwong, R. W., et al. (1998). Arabidopsis LEAFY COTYLEDON1 is sufficient to induce embryo development in vegetative cells. Cell 93, 1195-1205. doi: 10.1016/S00928674(00)81463-4

Manan, S., Ahmad, M. Z., Zhang, G., Chen, B., Haq, B. U., Yang, J., et al. (2017). Soybean LEC2 regulates subsets of genes involved in controlling the biosynthesis and catabolism of seed storage substances and seed development. Front. Plant Sci. 8:1604. doi: 10.3389/fpls.2017.01604

Mu, J., Tan, H., Zheng, Q., Fu, F., Liang, Y., Zhang, J., et al. (2008). LEAFY COTYLEDON1 is a key regulator of fatty acid biosynthesis in Arabidopsis. Plant Physiol. 148, 1042-1054. doi: 10.1104/pp.108.126342

Murashige, T., and Skoog, F. (1962). A revised medium for rapid growth and bioassays with tobacco tissue culture. Physiol. Plant 15, 473-497. doi: 10.1111/j. 1399-3054.1962.tb08052.x

Ohlrogge, J. B., and Browse, J. (1995). Lipid biosynthesis. Plant Cell 7, 957-970. doi: $10.1105 /$ tpc.7.7.957

Peng, F. Y., and Weselake, R. J. (2011). Gene coexpression clusters and putative regulatory elements underlying seed storage reserve accumulation in Arabidopsis. BMC Genomics 12:286. doi: 10.1186/1471-2164$12-286$

Salas, J. J., and Ohlrogge, J. B. (2002). Characterization of substrate specificity of plant FatA and FatB acyl-ACP thioesterases. Arch. Biochem. Biophys. 403, 25-34. doi: 10.1016/S0003-9861(02)00017-6

Sasaki, Y., and Nagano, Y. (2004). Plant acetyl-CoA carboxylase: structure, biosynthesis, regulation, and gene manipulation for plant breeding. Biosci. Biotechnol. Biochem. 68, 1175-1184. doi: 10.1271/bbb.68.1175

Shen, B., Allen, W. B., Zheng, P., Li, C., Glassman, K., Ranch, J., et al. (2010). Expression of ZmLEC1 and ZmWRI1 increases seed oil production in maize. Plant Physiol. 153, 980-987. doi: 10.1104/pp.110.157537

Shivani, P., Khushbu, P., Faldu, N., Thakkar, V., and Shubramanian, R. B. (2011). Extraction and analysis of Jatropha curcas L. seed oil. Afr. J. Biotechnol. 10, 18210-18213.

Shorrosh, B. S., Roesler, K. R., Shintani, D., van de Loo, F. J., and Ohlrogge, J. B. (1995). Structural analysis, plastid localization, and expression of the biotin carboxylase subunit of acetyl-coenzyme A carboxylase from tobacco. Plant Physiol. 108, 805-812. doi: 10.1104/pp.108.2.805

Stålberg, K., Ellerström, M., Josefsson, L., and Rask, L. (1993). Deletion analysis of a $2 \mathrm{~S}$ seed storage protein promoter of Brassica napus in transgenic tobacco. Plant Mol. Biol. 23, 671-683. doi: 10.1007/BF00021523

Stone, S. L., Kwong, L. W., Yee, K. M., Pelletier, J., Lepiniec, L., Fischer, R. L., et al. (2001). LEAFY COTYLEDON2 encodes a B3 domain transcription factor that induces embryo development. Proc. Natl. Acad. Sci. U.S.A. 98, 11806-11811. doi: $10.1073 /$ pnas. 201413498 
Tan, H., Yang, X., Zhang, F., Zheng, X., Qu, C., Mu, J., et al. (2011). Enhanced seed oil production in canola by conditional expression of Brassica napus LEAFY COTYLEDON1 and LEC1-LIKE in developing seeds. Plant Physiol. 156, 1577-1588. doi: 10.1104/pp.111.175000

Tang, G. Y., Wei, L. Q., Liu, Z. J., Bi, Y. P., and Shan, L. (2012). Ectopic expression of peanut acyl carrier protein in tobacco alters fatty acid composition in the leaf and resistance to cold stress. Biol. Plantarum 56, 493-501. doi: 10.1007/s10535012-0057-7

Thelen, J. J., and Ohlrogge, J. B. (2002). Metabolic engineering of fatty acid biosynthesis in plants. Metab. Eng. 4, 12-21. doi: 10.1006/mben.2001. 0204

Tovey, E. R., and Baldo, B. A. (1987). Comparison of semi-dry and conventional tank-buffer electrotransfer of proteins from polyacrylamide gels to nitrocellulose membranes. Electrophoresis 8, 384-387. doi: 10.1002/elps. 1150080904

Troncoso-Ponce, M. A., Kilaru, A., Cao, X., Durrett, T. P., Fan, J., Jensen, J. K., et al. (2011). Comparative deep transcriptional profiling of four developing oilseeds. Plant J. 68, 1014-1027. doi: 10.1111/j.1365-313X.2011.04751.x

USDA. (2018). Table01: Major Oilseeds: World Supply and Distribution (Commondity View). Available at: http://apps.fas.usda.gov/psdonline/ps dReport.aspx?hidReportRetrievalName $=$ Table $+01 \% 3 a+$ Major+Oilseeds $\% 3 a+$ World+Supply+and+Distribution+(Commodity+View $) \&$ hidReportRetrievalI $\mathrm{D}=531$ \&hidReportRetrievalTemplateID $=5$

Voelker, T., and Kinney, A. J. (2001). Variations in the biosynthesis of seed-storage lipids. Annu. Rev. Plant Physiol. Plant Mol. Biol. 52, 335-361. doi: 10.1146/ annurev.Arplant.52.1.335

Wallis, J. G., and Browse, J. (2002). Mutants of Arabidopsis reveal many roles for membrane lipids. Prog. Lipid Res. 41, 254-278. doi: 10.1016/S0163-7827(01) 00027-3

Weselake, R. J., Taylor, D. C., Rahman, M. H., Shah, S., Laroche, A., McVetty, P. B., et al. (2009). Increasing the flow of carbon into seed oil. Biotechnol. Adv. 27, 866-878. doi: 10.1016/j.biotechadv.2009.07.001
Woodfield, H. K., Sturtevant, D., Borisjuk, L., Munz, E., Guschina, I. A., Chapman, K., et al. (2017). Spatial and temporal mapping of key lipid species in Brassica napus seeds. Plant Physiol. 173, 1998-2009. doi: 10.1104/pp.16. 01705

Yu, M., Liu, F., Zhu, W., Sun, M., Liu, J., and Li, X. (2015). New features of triacylglycerol biosynthetic pathways of peanut seeds in early developmental stages. Funct. Integr. Genomics 15, 707-716. doi: 10.1007/s10142-015-0447-y

Zheng, L., Shockey, J., Guo, F., Shi, L., Li, X., Shan, L., et al. (2017). Discovery of a new mechanism for regulation of plant triacylglycerol metabolism: the peanut diacylglycerol acyltransferase-1 gene family transcriptome is highly enriched in alternative splicing variants. J. Plant Physiol. 219, 62-70. doi: 10.1016/j.jplph. 2017.09.009

Zou, J., Katavic, V., Giblin, E. M., Barton, D. L., MacKenzie, S. L., Keller, W. A., et al. (1997). Modification of seed oil content and acyl composition in the brassicaceae by expression of a yeast sn-2 acyltransferase gene. Plant Cell 9, 909-923. doi: 10.1105/tpc.9.6.909

Zou, J., Wei, Y., Jako, C., Kumar, A., Selvaraj, G., and Taylor, D. C. (1999). The Arabidopsis thaliana TAG1 mutant has a mutation in a diacylglycerol acyltransferase gene. Plant J. 19, 645-653. doi: 10.1046/j.1365-313x.1999. 00555.x

Conflict of Interest Statement: The authors declare that the research was conducted in the absence of any commercial or financial relationships that could be construed as a potential conflict of interest.

Copyright (c) 2018 Tang, Xu, Ma, Wang, Liu, Wan and Shan. This is an open-access article distributed under the terms of the Creative Commons Attribution License (CC BY). The use, distribution or reproduction in other forums is permitted, provided the original author(s) and the copyright owner are credited and that the original publication in this journal is cited, in accordance with accepted academic practice. No use, distribution or reproduction is permitted which does not comply with these terms. 